\section{Determinantes psicosociales de la intención de donación de órganos en una muestra chilena}

\author{
ISIDORA DOGGENWEILER ${ }^{\mathrm{a}}$, ELIANA GUIC $^{\mathrm{b}}$
}

\section{Psychosocial determinants of organ donation among Chilean university students}

Background: Organ donation (OD) is a complex process that among other factors, depends on the consent of the potential donor's family. Previous evidences have shown that the intention of a behavior predicts that behavior in the future. Aim: To study the effect of believes, worries, knowledge and attitudes on the intention of $O D$ after own death as well as OD of a deceased relative. Material and Methods: A total of 3,297 Chilean university students responded to a survey about the intention to donate organs through a web link. We conducted a path analysis and a structural equation technique was used. The model explained more than $50 \%$ of the variance of the dependent variables. Results: The intention of respondents to donate their own organs or those of family members after death were predicted by attitude toward OD, social influences and family discussion. Attitude was the main predictor of the model, which is determined by the negative effect of bodily apprehensions and the positive effect of knowledge about brain death. Conclusions: Attitude, knowledge and concerns are the main determinants of donation intention. The results prove the validity of the Theory of Reasoned Action as a theoretical model to explain the intentions of OD.

(Rev Med Chile 2014; 142: 27-33)

Key words: Attitude; Tissue and organ procurement; Transplantation.

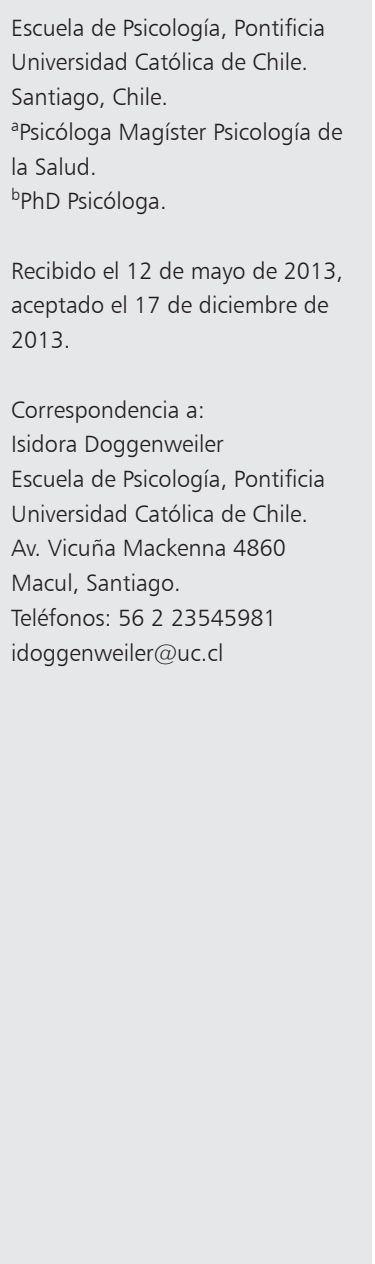
Universidad Católica de Chile. Santiago, Chile. apsicóloga Magíster Psicología de la Salud. Psicóloga.

Recibido el 12 de mayo de 2013 ,
aceptado el 17 de diciembre de
2013.
Correspondencia a:
Isidora Doggenweiler
Escuela de Psicología, Pontificia
Universidad Católica de Chile.
Av. Vicuña Mackenna 4860
Macul, Santiago.
Teléfonos: 56223545981
idoggenweiler@uc.cl

$\mathrm{E}$ l creciente déficit de la donación de órganos, debido al abismo entre la escasa disponibilidad y las necesidades de la población, es reconocido a nivel mundial. Según los datos aportados por la Corporación de Trasplante (CT), actualmente en Chile hay 1.370 personas en espera de un trasplante de órgano ${ }^{1}$, cifra que se enfrenta a una realidad de 6,6 donantes por millón de habitantes (p.m.p), número restringido en comparación al promedio de 35,3 donantes p.m.p de España, país líder en donación ${ }^{2}$.

La donación de órganos es un proceso complejo que depende de la disponibilidad de condiciones técnicas (infraestructura y capital humano) para la pesquisa y mantenimiento del potencial donante. Esto, en conjunto con la participación directa de

la sociedad, representada por los familiares del potencial donante, que debe otorgar la autorización para realizar la extracción de órganos de un familiar fallecido. Independiente del tipo de consentimiento (presunto o explícito) y pese a la aprobación en vida del fallecido, siempre es la familia la convocada a tomar la decisión final.

Los esfuerzos enfocados a mejorar las falencias en el sistema de pesquisa y manejo de potenciales donantes de órganos y el traspaso de las responsabilidades operativas de la Corporación de Trasplante al Ministerio de Salud, han significado gestiones beneficiosas para el modelo de donación chileno $^{3}$. Sin embargo, el impacto negativo representado por el rechazo familiar a la solicitud de donación, actualmente asciende al 50\% del total 
de las solicitudes, superando el promedio histórico nacional de $35 \%{ }^{3}$.

El fracaso de las iniciativas enfocadas a reducir el impacto de la negativa familiar, como la Ley 20.143 del "Donante Universal"3, muestran la necesidad de avanzar en una comprensión más profunda de la dimensión psicosocial de la donación de órganos. El presente estudio tiene como objetivo conocer el efecto que tienen determinadas creencias, preocupaciones, conocimientos y actitudes en la intención de convertirse en donante después de la muerte y en la intención de donar los órganos de un familiar fallecido. Esto con el propósito de aportar información válida y confiable, que sirva al diseño de estrategias que logren aumentar el índice de donación en el país.

\section{El modelo de la acción razonada y de donación de órganos}

Desde la perspectiva teórica de la cognición social, el presente estudio construye un modelo predictivo de las intenciones de realizar conductas de donación, basado en la adaptación y extensión de la teoría de la acción razonada (TRA) ${ }^{7}$. Este modelo teórico se ha utilizado en múltiples trabajos en los que se busca a los determinantes de las conductas de donación de órganos ${ }^{6,8-14}$.

La TRA es un modelo utilizado para la comprensión y predicción de la conducta individual y voluntaria, a la cual se le atribuye como su principal y más directo determinante la intención. Las intenciones conductuales se originan en creencias y conocimientos que ejercen su influencia a través de las actitudes y las normas subjetivas. Las actitudes se entienden como el resultado de un juicio valorativo hacia un objeto o conducta, y las normas subjetivas representan la influencia que ejercen sobre el individuo la presión y expectativa social relacionada con la ejecución u omisión de determinada conducta.

Los estudios han demostrado consistentemente que existe una actitud favorable de las personas en diferentes países hacia el concepto general de donación de órganos ${ }^{4,6,8,11}$. Estas actitudes favorables, sumadas al efecto percibido de normas subjetivas sostenedoras de las conductas de donación, promueven intenciones positivas hacia la donación de órganos propios y de los familiares, así como también hacia la discusión familiar sobre la temática de donación $n^{6,11,13}$. Una de las principales causas en el mundo de negativa familiar a la solicitud de donación, es por desconocimiento de la postura del familiar que ha fallecido ${ }^{3,6}$. Un porcentaje cercano, o inferior al $50 \%$ de las personas conocen la intención de sus familiares y han comunicado las propias ${ }^{4,6}$.

Los principales hallazgos de las investigaciones que utilizan modelos extendidos de la TRA, evidencian el impacto que provocan las temáticas que rodean el fenómeno de la donación de órganos, como la noción de muerte y de cuerpo cadavérico. Pensar en la donación de órganos necesariamente implica pensar en la muerte propia o de los cercanos y además obliga a decidir aspectos relacionadas con la manipulación del cadáver. Los estudios muestran como estas temáticas producen rechazo, ansiedad y miedo ${ }^{12,17}$.

Se ha llamado aprensiones corporales a factores no cognitivos, relacionados con las preocupaciones respecto a la manipulación del cadáver del donante y las consecuencias de la extracción de órganos. Estas preocupaciones son emociones poco placenteras, como miedo o ansiedades específicas a la desfiguración corporal. Pueden estar arraigadas en creencias religiosas o supersticiones ${ }^{12,13}$. El proceso de extracción de órganos puede ser percibido como una verdadera mutilación que deja el cuerpo desfigurado con cortes y cicatrices $s^{5,13,18}$. También es común la creencia de que el cuerpo debe permanecer intacto para el entierro, siendo la extracción de órganos una falta de respeto hacia el fallecido ${ }^{9,10,11,19}$. Estas preocupaciones representan motivos frecuentes de rechazo a la donación ${ }^{5,13,18}$.

El diagnóstico de muerte encefálica (ME),representa un cambio radical de la concepción de muerte, por lo que se comprende su efecto perturbador y las resistencias y rechazo que ha generado en la población $5,11,17,19$. Un gran número de personas que se declaran como no donantes, creen que los pacientes diagnosticados con ME no están realmente muertos ${ }^{9,17}$. También es frecuente que el diagnóstico de ME se confunda con estados como el coma y el estado vegetativo persistente ${ }^{17}$. Estas confusiones y errores, sumados también a temores relacionados con la posibilidad de que el paciente con ME pueda sentir dolor durante el proceso de extracción de órganos, constituyen una de las principales causas descritas de negativa hacia las conductas de donación ${ }^{9,11}$.

Por último, la discusión familiar considerada como un aspecto integral de la toma de decisión sobre la donación de órganos, también se ve afec- 
tada por la connotación de tabú que acompañan las temáticas relacionadas con la muerte y el cadáver en la sociedad actual ${ }^{14,15,20}$. Las creencias de que hablar de la muerte trae "mala suerte" ${ }^{12,14}$ y el temor asociado a las reacciones de los familiares al plantear el tema, contribuye a que las personas eviten involucrarse en este tipo de conversaciones $^{11,14,20,21}$.

En base a los hallazgos de las investigaciones previas, el presente trabajo elabora un modelo predictivo que incorpora como variables distales una medición de conocimientos específicos sobre ME y de las aprensiones corporales relacionadas con la manipulación del cuerpo cadavérico. Los constructos clásicos de la TRA (actitudes y normas subjetivas) en conjunto con una medición de la disposición a la discusión familiar, conforman las variables mediadoras entre las variables dependientes (intenciones de donación) y los predictores distales.

\section{Material y Método}

\section{Participantes}

La muestra está compuesta por 3.297 estudiantes universitarios de la Pontificia Universidad Católica de Chile, 35,6\% hombres y 64,4\% mujeres, con un promedio de 22, 6 años de edad y una desviación estándar de 6,9 años.

\section{Procedimiento}

Utilizando internet como plataforma, se envió una invitación al correo electrónico de todos los alumnos regulares de los programas de estudio impartidos en la Pontificia Universidad Católica de Chile. Los interesados en participar del estudio accedieron a la encuesta mediante un enlace web, que estuvo disponible durante septiembre y octubre del año 2011. Como requisito, los participantes debían aprobar un consentimiento informado confeccionado a partir de las normas éticas exigidas internacionalmente y aprobado por el comité de ética de la Escuela de Psicología de dicha institución.

\section{Medición}

Se elaboró un instrumento de auto reporte a partir de mediciones utilizadas por investigaciones sobre donación basadas en la TRA. Las escalas que componen las variables independientes fueron adaptadas a partir de las mediciones utilizadas por Morgan et al. ${ }^{11}$ y Stephenson et al. ${ }^{13} \mathrm{y} \mathrm{Hyde}^{22}$. El instrumento fue sometido a una primera etapa de validación preliminar. En la Tabla 1 se muestran los ítems e información descriptiva de las variables dependientes e independientes.

\section{Análisis estadístico}

El modelo teórico se prueba mediante un análisis de sendero (path analisys) con un modelo identificado justo, técnica que permite hacer una evaluación de las relaciones entre las variables en conjunto ajustadas al modelo teórico propuesto en la introducción. Para el desarrollo de las ecuaciones estructurales que implica el análisis de senderos se utilizó el paquete de análisis estadístico MPlus 5.0. También se realiza un análisis descriptivo general de los resultados.

\section{Resultados}

En primer lugar se muestra el análisis descriptivo de las variables dependientes, posteriormente se exponen las relaciones bivariadas del set de variables incluidos en el modelo. (Tabla 2). Finalmente se describen los resultados del modelo de ecuación estructural y sus efectos directos e indirectos que se desprenden del modelo.

En general, los estudiantes presentaron una intención positiva a donar los órganos propios después de la muerte $(\mathrm{M}=5.37 / 6)$ y a donar los órganos de familiares fallecidos $(\mathrm{M}=4.91 / 6)$. Las relaciones bivariadas mostraron cómo las variables dependientes estuvieron relacionadas significativamente con todas las variables independientes.

El modelo resultante del análisis de senderos y la ecuación estructural, explica el 65\% (R2) de la varianza de la intención de donar los propios órganos y el 51\% (R2) de la varianza de la intención de donar los órganos de un familiar.

Los constructos TRA (normas subjetivas y actitudes), en conjunto con la discusión familiar, se comportaron como predictores positivos de las variables dependientes. La actitud fue el predictor más importante del modelo, tanto para la intención de donar los órganos propios $(\beta=$, $72, \mathrm{p}<, 01)$, como para la intención a donar los órganos de familiares $(\beta=, 49, \mathrm{p}<, 01)$.

Los resultados del comportamiento de los predictores en el modelo se muestran en la Figura 1. 
Tabla 1. Descripción de la medición de las variables dependientes e independientes

\begin{tabular}{|c|c|c|c|}
\hline $\begin{array}{l}\text { Variables } \\
\text { dependientes }\end{array}$ & Ítem & Respuesta & Descriptivos \\
\hline $\begin{array}{l}\text { Intención de donar } \\
\text { órganos propios }\end{array}$ & $\begin{array}{l}\text { ¿Estás dispuesto a que tus órganos sean } \\
\text { donados después de tu muerte? }\end{array}$ & $\begin{array}{l}\text { 1. No estoy dispuesto } \\
\text { 2. } \\
\text { 3. } \\
\text { 4. } \\
\text { 5. } \\
\text { 6. Totalmente dispuesto }\end{array}$ & $\begin{array}{l}\text { Media }=5,37 \\
\mathrm{DE}=1,28\end{array}$ \\
\hline $\begin{array}{l}\text { Intención de donar } \\
\text { órganos de un familiar }\end{array}$ & $\begin{array}{l}\text { Si un familiar tuyo falleciera ¿Estás dispuesto } \\
\text { a donar sus órganos? }\end{array}$ & $\begin{array}{l}\text { 1. No estoy dispuesto } \\
\text { 2. } \\
\text { 3. } \\
\text { 4. } \\
\text { 5. } \\
\text { 6. Totalmente dispuesto }\end{array}$ & $\begin{array}{l}\text { Media }=4,91 \\
\mathrm{DE}=1,37\end{array}$ \\
\hline
\end{tabular}

\begin{tabular}{|c|c|c|c|}
\hline $\begin{array}{l}\text { Variables } \\
\text { independientes }\end{array}$ & Variable & Operacionalización & Descriptivos \\
\hline $\begin{array}{l}\text { Conocimientos sobre } \\
\text { muerte encefálica }\end{array}$ & $\begin{array}{l}\text { Indica la respuesta correcta: } \\
\text { - ¿Una persona con muerte encefálica se } \\
\text { puede recuperar de su estado? } \\
\text { - ¿La gente con muerte encefálica puede } \\
\text { sentir dolor cuando sus órganos son ex- } \\
\text { traídos? } \\
\text { Una paciente al que se le ha diagnosticado } \\
\text { muerte encefálica está? (muerto, como si } \\
\text { estuviera muerto o vivo) }\end{array}$ & $\begin{array}{l}\text { Suma de respuestas correctas } \\
\text { Min. }=0 \\
\text { Máx. }=3\end{array}$ & $\begin{array}{l}\text { Media }=1,08 \\
\mathrm{DE}=0,92\end{array}$ \\
\hline Aprensiones corporales & $\begin{array}{l}\text { - Indica el grado de acuerdo con cada una } \\
\text { de las siguientes afirmaciones: } \\
\text { - Me preocupa que va a ocurrir con mi cuer- } \\
\text { po después que esté muerto* } \\
\text { - Me preocupa que el cuerpo pueda quedar } \\
\text { con alguna cicatriz o desfigurado tras la } \\
\text { extracción de órgano }\end{array}$ & $\begin{array}{l}\text { 1. Muy en desacuerdo } \\
\text { 2. . } \\
\text { 3. . } \\
\text { 4. . } \\
\text { 5. . } \\
\text { 6. Muy de acuerdo }\end{array}$ & $\begin{array}{l}\text { Media }=2,02 \\
\mathrm{DE}=1,06 \\
\text { Correlación } \\
\text { policorica }=, 25^{* *}\end{array}$ \\
\hline $\begin{array}{l}\text { Actitud hacia la } \\
\text { donación de órganos }\end{array}$ & $\begin{array}{l}\text { Marca el número que mejor represente tu } \\
\text { postura: } \\
\text { ¿Cuál es tu postura hacia la donación de } \\
\text { órganos? }\end{array}$ & $\begin{array}{l}\text { 1. Estoy en contra } \\
\text { 2. . } \\
\text { 3. . } \\
\text { 4. . } \\
\text { 5. . } \\
\text { 6. Estoy a favor }\end{array}$ & $\begin{array}{l}\text { Media }=5,48 \\
D E=1,07\end{array}$ \\
\hline Discusión familiar & $\begin{array}{l}\text { Marca el número que mejor describa tu } \\
\text { disposición: } \\
\text { ¿Estás dispuesto a discutir con tu familia, } \\
\text { durante este mes, sobre donación de ór- } \\
\text { ganos? }\end{array}$ & $\begin{array}{l}\text { 1. No estoy dispuesto } \\
\text { 2. . } \\
\text { 3. . } \\
\text { 4. . } \\
\text { 5. . } \\
\text { 6. Totalmente dispuesto }\end{array}$ & $\begin{array}{l}\text { Media }=5,18 \\
D E=1,32\end{array}$ \\
\hline Normas subjetivas & $\begin{array}{l}\text { Indica el número que mejor te represente: } \\
\text { - La mayoría de las personas que son im- } \\
\text { portantes para mí piensan que debo ser un } \\
\text { donante. } \\
\text { - La mayoría de las personas que son im- } \\
\text { portantes para mí están dispuestos a ser } \\
\text { donantes. }\end{array}$ & $\begin{array}{l}\text { 1. Muy en desacuerdo } \\
\text { 2. . } \\
\text { 3. . } \\
\text { 4. . } \\
\text { 5. . } \\
\text { 6. Muy de acuerdo }\end{array}$ & $\begin{array}{l}\text { Media }=4,28 \\
\mathrm{DE}=1,33 \\
\text { Correlación } \\
\text { policorica }=, 80^{* *}\end{array}$ \\
\hline
\end{tabular}


Tabla 2. Relaciones bivariadas

\begin{tabular}{|c|c|c|c|c|c|c|}
\hline & 1 & 2 & 3 & 4 & 5 & 6 \\
\hline \multicolumn{7}{|l|}{ 1. Intención de DO propios } \\
\hline 2. Intención de DO de un familiar & $0,73 * * *$ & & & & & \\
\hline 3. Aprensiones corporales & $-0,19 * * *$ & $-0,26 * * *$ & & & & \\
\hline 4. Normas Subjetivas & $0,44^{* * *}$ & $0,50 * * *$ & $-0,19 * * *$ & & & \\
\hline 5. Actitud & $0,80 * * *$ & $0,64 * * *$ & $-0,19 * * *$ & $0,41 * * *$ & & \\
\hline 6. Conocimiento sobre ME & $0,14^{* * *}$ & $0,19 * * *$ & $-0,15^{* * *}$ & $0,15^{* * *}$ & $0,15^{* * *}$ & \\
\hline 7. Discusión familiar & $0,31 * * *$ & $0,33^{* * *}$ & $-0,15^{* * *}$ & $0,32 * * *$ & $0,25^{* * *}$ & $0,08^{* * *}$ \\
\hline
\end{tabular}

Nota ${ }^{* *}=p<0,01$.

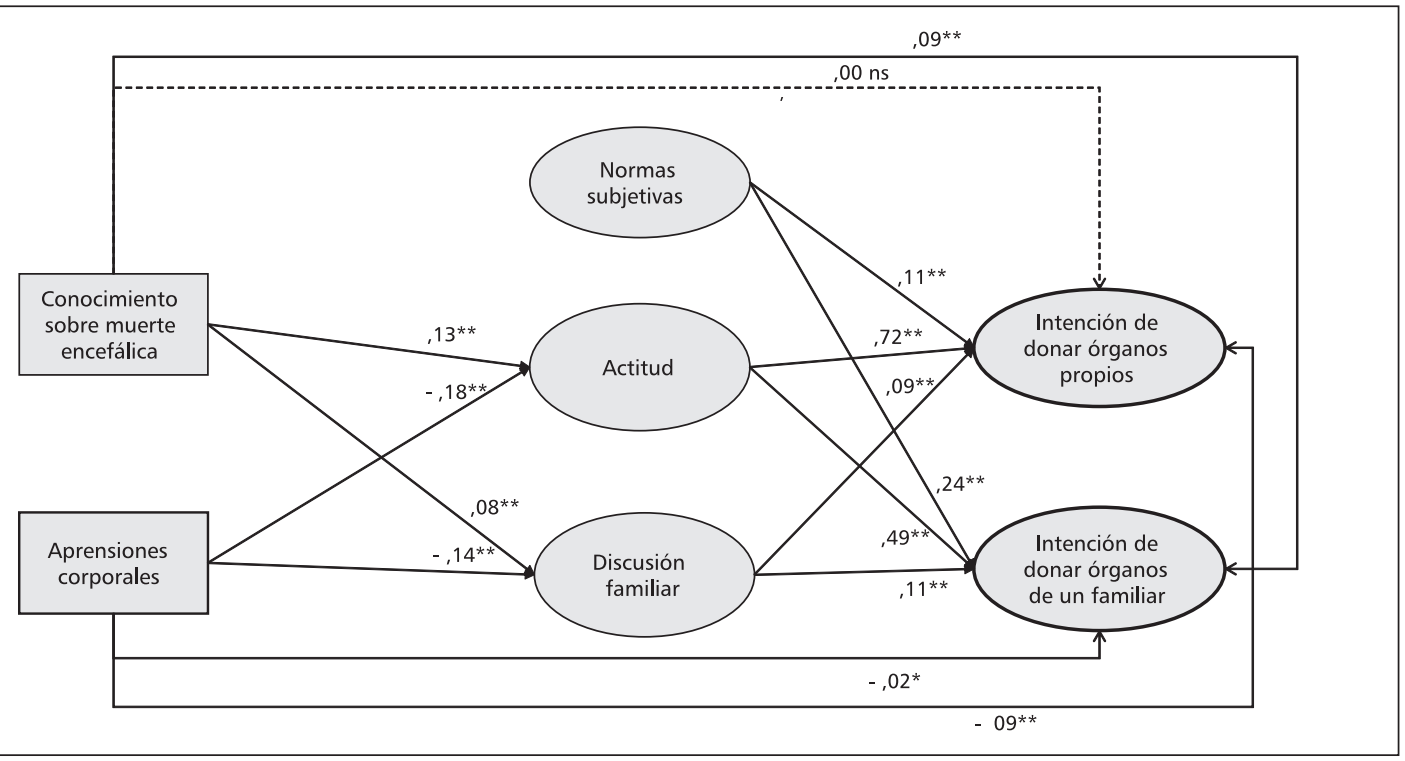

Figura 1. Resultados modelo ecuación estructural. Nota: ${ }^{*}=p<, 05 ;{ }^{*}=p<, 01$.

Las aprensiones corporales mostraron un efecto negativo directo en la intención de donar los órganos de los familiares $(\beta=-, 02, p<, 05)$ y en la intención de donar los propios órganos $(\beta$ $=-.09, \mathrm{p}<, 01)$ y negativo indirecto mediante la actitud $(\beta=-, 14, \mathrm{p}<, 01)$ y la discusión familiar $(\beta=-, 14, \mathrm{p}<, 01)$. Los conocimientos sobre ME mostraron un efecto positivo directo sólo en la intención de donar los órganos de familiares $(\beta$ $=, 09, \mathrm{p}<, 01)$ y positivo indirecto mediante la actitud $(\beta=-, 13, \mathrm{p}<, 01)$ y la discusión familiar $(\beta=-, 8, \mathrm{p}<, 01)$.

\section{Discusión}

El presente estudio muestra que una actitud positiva a la donación, influenciada por preocupaciones en relación al cuerpo y conocimientos sobre muerte encefálica. Estas variables predicen la intención de donación de órganos, así como también las influencias sociales y conversación con la familia. Este es el primer trabajo en una muestra chilena que presenta un modelo psicosocial explicativo basado en evidencias, para comprender la donación de órganos. Por lo tanto, provee información interesante para el diseño de intervenciones que favorezcan la donación de órganos en el país.

El presente estudio muestra que ambas intenciones de donación, de órganos propios y de un familiar, son predichas por las mismas variables, es decir actitud, discusión familiar y normas 
subjetivas. La actitud representó el predictor más importante del modelo, el que está determinado por el efecto significativo y negativo de las aprensiones corporales y el efecto positivo de los conocimientos sobre ME. Se concluye la relevancia de la actitud favorable a la donación y el efecto sobre ésta de los conocimientos y preocupaciones, como determinantes de las intenciones de donación. Las normas subjetivas representan la influencia social, o sea también importa lo que piensan los demás en relación a la donación. Los resultados comprueban la validez de la TRA como modelo teórico para explicar las intenciones de donación y su efectividad para estudiar los determinantes de la intención de donación. No se espera que las normas subjetivas cumplan una función mediadora, así como la actitud y la discusión familiar, ya que no es conceptualmente coherente. Por esta razón tampoco hay registros previos de la relación entre normas subjetivas y las variables distales propuestas en nuestro modelo.

La discusión familiar, variable que no forma parte del modelo TRA, pero es incluida como variable mediadora, actúa como un propulsor significativo de ambas intenciones estudiadas. Es interesante constatar que la discusión familiar es afectada negativamente por las aprensiones corporales. Al parecer la ansiedad y rechazo que producen las temáticas relacionadas con el cadáver y la muerte efectivamente hacen que las personas eviten involucrarse en este tipo de conversaciones, tal como se señala en las investigaciones analizadas ${ }^{11,16,20,21}$. Otra explicación que puede revelar el efecto negativo de las aprensiones corporales es el temor a las posibles reacciones de los familiares al abordar temáticas que tienen una connotación de tabú como lo es la muerte y el cadáver ${ }^{11,14,20,21}$.

Las aprensiones corporales, destacan también por su efecto negativo y significativo en ambas intenciones, es importante señalar que el efecto es mayor en la intención de donar los órganos propios. Es posible pensar que cuando se trata del propio cuerpo, las preocupaciones relacionadas con la manipulación del cuerpo y las consecuencias de la extracción, cobran mayor relevancia.

Los resultados muestran cómo las personas que cuentan con conocimientos claros y certeros sobre la ME, tienden a manifestar una actitud más favorable hacia la donación y una mayor disposición hacia la discusión familiar. Es interesante el efecto significativo positivo y directo que presentan estos conocimientos hacia la intención de donar los órganos de un familiar, pero no hacia la intención de donar los propios órganos. Esta diferencia, hace aun más evidente la importancia que puede tener comprender el diagnóstico de $\mathrm{ME}$ (como una condición única e irreversible), para los familiares que se enfrentan a una solicitud de donación en un momento de duelo.

El presente estudio provee información relevante para el diseño de estrategias de intervención orientadas a aumentar el índice de donación en el país. La actitud es susceptible de ser intervenida a través de la educación sobre el diagnóstico de muerte encefálica y la clarificación de las preocupaciones relacionadas con las aprensiones corporales. El objetivo de la educación tendría que ser el de disminuir las ansiedades y temores que surgen a raíz del desconocimiento y la confusión, considerando la ansiedad y rechazo que producen estas temáticas. Una vez que se cumpla con esta etapa educativa, fomentar la discusión familiar contribuiría a vencer resistencias y facilitar la socialización de los conocimientos relacionados con el proceso de donación. Lo que al mismo tiempo puede influenciar positivamente el efecto de las normas subjetivas.

Por último, es importante mencionar que los resultados de este estudio se limitan a una muestra particular, por lo que es necesario considerar las restricciones de su aplicabilidad a la población general.

\section{Referencias}

1. Corporación del Trasplante. (2012). Lista de espera en Chile. Extraído el 7 de noviembre de 2012, del sitio web la Corporación del Trasplante: http://www.trasplante.cl

2. International Registry in Organ Donation and Transplantation (2012). Second report data. 2011. Extraído el 22 de noviembre de 2012, del sitio web la Corporación de Trasplante: http://www.trasplante.cl/noticias/ internacionales/205-2o-reporte-internacional-donacion-y trasplantes-de-organos.html

3. Corporación del Trasplante. (2012). Memoria 2011. Extraído el 7 de noviembre de 2012, del sitio web la Corporación del Trasplante: http://www.trasplante.cl/ utilidades/biblioteca/category/2-memorias.html

4. Caballer A. La actitud de la donación de órganos en la población española: Análisis mediante regresión logística multinivel [Versión electrónica]. 2001. Tesis para optar al Título de Doctor en Psicología. Depar- 
tamento de Psicología Evolutiva, Educativa, Social y Metodológica, Universidad Jaume I de Castellón. Castellón, España. http://repodoc.uji.es/xmlui/bitstream/ handle/10803/10519/caballer.pdf?...1.

5. Ghorbani F, Khoddami-Vishteh HR, Ghobadi O, Shafaghi S, Louyeh AR, Najafizadeh K. Causes of family refusal for organ donation [Versión electrónica]. Transplantation Proceedings 2011; 43: 405-6. doi:10.1016/j. transproceed.2011.01.031.

6. Hyde MK, White KM. To be a donor or not to be? Applying an extended theory of planned behavior to predict posthumous organ donation intentions [Versión electrónica]. Journal of Applied Social Psychology 2009; 39 (4): 880-900. doi:10.1111/j.559-1816.2009.00464.x

7. Fishbein M, Ajzen I. Belief, attitude, intention, and behavior: An introduction in theory and research, Reading, MA 1975: Addison-Wesley.

8. Feeley TH. College students' knowledge, attitudes, and behaviors regarding organ donation: An integrated review of the literature [Versión electrónica]. Journal of Applied Social Psychology 2007; 37 (2): 243-71. doi: 10.1111/j.0021-9029.2007.00159.x

9. Morgan SE, Miller JK. Beyond the organ donor card: The effect of knowledge, attitudes, and values on willingness to communicate about organ donation to family members [Versión electrónica]. Health Communications 2002; 14 (1): 121-34. doi: 10.1207/S15327027 HC1401_6.

10. Morgan SE, Miller JK. Communicating about gifts of life: The effect of knowledge, attitudes, and altruism on behavior and behavioral intentions regarding organ donation [Versión electrónica]. Journal of Applied Communication Research 2002; 30 (2): 163-78. doi: 10. 1080/00909880216580.

11. Morgan SE, Miller JK, Arasaratnam LA. Similarities and difference between african americans' and european american's attitude, knowledge, and willingness to communicate about organ donation [Versión electrónica]. Journal of Applied Psychology 2003; 33 (4): 693-715. doi: 10.1111/j.1559-1816.2003.tb01920.x

12. Morgan SE, Stephenson MT, Harrison TR, Afifi WA, Long SD. Facts versus 'fellings'. How rational is the decision to become an organ donor? [Versión electrónica]. Journal of Health Psychology 2009; 13 (5): 664-58. doi: 10.1177/1359105308090936.

13. Stephenson MT, Morgan SE, Roberts-Pérez SD, Harrison T, Afifi W, Long SD. The role of religiosity, religions norms, subjective norms, and bodily integrity in signing an organ donor card. [Versión electrónica]. Health Communication 2008; 23: 436-47. doi: 10.1080/
10410230802342119.

14. Morse C, Afifi WA, Morgan SE, Stephenson MT, Reichert T, Harrison TR, Long SD. Religiosity, anxiety, and discussions about organ: Understanding a complex system of associations [Versión electrónica]. Health Communication 2009; 24: 156-64. doi: $10.1080 / 10410230802676755$.

15. Guerra R. Donación de órganos: Comprensión y significado [Versión electrónica]. Tesis para Optar al Título de Sociólogo 2005. Universidad de Chile, Facultad de Ciencias Sociales, Escuela de Sociología, Santiago, Chile. http://www.tesis.uchile.cl/tesis/uchile/2005/guerra_r/ html/index-frames.html

16. Kerridge I, Saul P, Lowe M, McPhee J, Williams D. Death, dying and donation: Organ transplantation and the diagnosis of death [Versión electrónica]. Journal of Medical Ethics 2002; 28 (2): 89-94. doi: 10.1136/ jme.28.2.89.

17. Siminoff LA, Burant C, Younger SJ. Death and organ procurement: public beliefs and attitudes [Versión electrónica]. Social Science and Medicine 2004; 59 (11): 2325-34. doi: S0277953604001601.

18. Morgan SE. Many facets of reluctance: African americans and the decision (not) to donate organs [Versión electrónica]. Journal of the National Medical Association 2006; 98 (5): 695-703. Pub Med Data base http:// www.ncbi.nlm.nih.gov/pmc/articles/PMC2569263/ \#referencesec

19. Ohnuki-Tierney E. Brain death and organ transplantation: Cultural bases of medical technology [Versión electrónica]. Current Anthropology 1994; 35 (3): 23354. http://www.jstor.org/stable/2744197

20. Afifi WA, Morgan SE, Morse C, Stephenson MT, Harrison TR, Reichert T, Long D. Examining the decision to talk about organ donation: A test of the theory of motivated information management [Versión electrónica]. Communication Monographs 2006; 73 (2): 188-215. doi: 10.1080/03637750600690700.

21. Smith SW, Massi LL, Kopfman JE, Yoo J, Morrison K. Predictors of engaging in family discussion about organ donation and getting organ donor card witnessed [Versión electrónica]. Health Communication 2008; 23 (2): 142-52. doi: 10.1080/10410230801968104.

22. Hyde M. Determining the psychosocial predictors of living, living-relates, and posthumous organ donation [Version electrónica]. Thesis for the degree of Doctor of Philosophy 2009. Queensland University of Technology School of Psychology and Counseling Brisbane, Australia. http://eprints.qut.edu.au/29724/2/Melissa_Hyde_Thesis.pdf 